\title{
Absorber Materials for Transition-Edge Sensor X-ray Microcalorimeters
}

Arrays of superconducting transition-edge sensors (TES) can provide high spatial and energy resolution necessary for $\mathrm{x}$-ray astronomy. High quantum efficiency and uniformity of response can be achieved with a suitable absorber material, in which absorber x-ray stopping power, heat capacity, and thermal conductivity are relevant parameters. Here we compare these parameters for bismuth and gold. We have fabricated electroplated gold, electroplated gold/electroplated bismuth, and evaporated gold/evaporated bismuth $8 \times 8$ absorber arrays and find that a correlation exists between the residual resistance ratio (RRR) and thin film microstructure. This finding indicates that we can tailor absorber material conductivity via microstructure alteration, so as to permit absorber thermalization on timescales suitable for high energy resolution $\mathrm{x}$-ray microcalorimetry. We show that by incorporating absorbers possessing large grain size, including electroplated gold and electroplated gold/electroplated bismuth, into our current $\mathrm{Mo} / \mathrm{Au} \mathrm{TES}$, devices with tunable heat capacity and energy resolution of $2.3 \mathrm{eV}$ (gold) and $2.1 \mathrm{eV}$ (gold/bismuth) FWHM at $6 \mathrm{keV}$ have been fabricated.

A. -D. Brown, S. R. Bandler, R. Brekosky, J. A. Chervenak, E. Figueroa-Feliciano, F. Finkbeiner, N. Iyomoto, R.L. Kelley, C.A. Kilbourne, F.S. Porter, S. Smith, T. Saab, J. Sadleir 


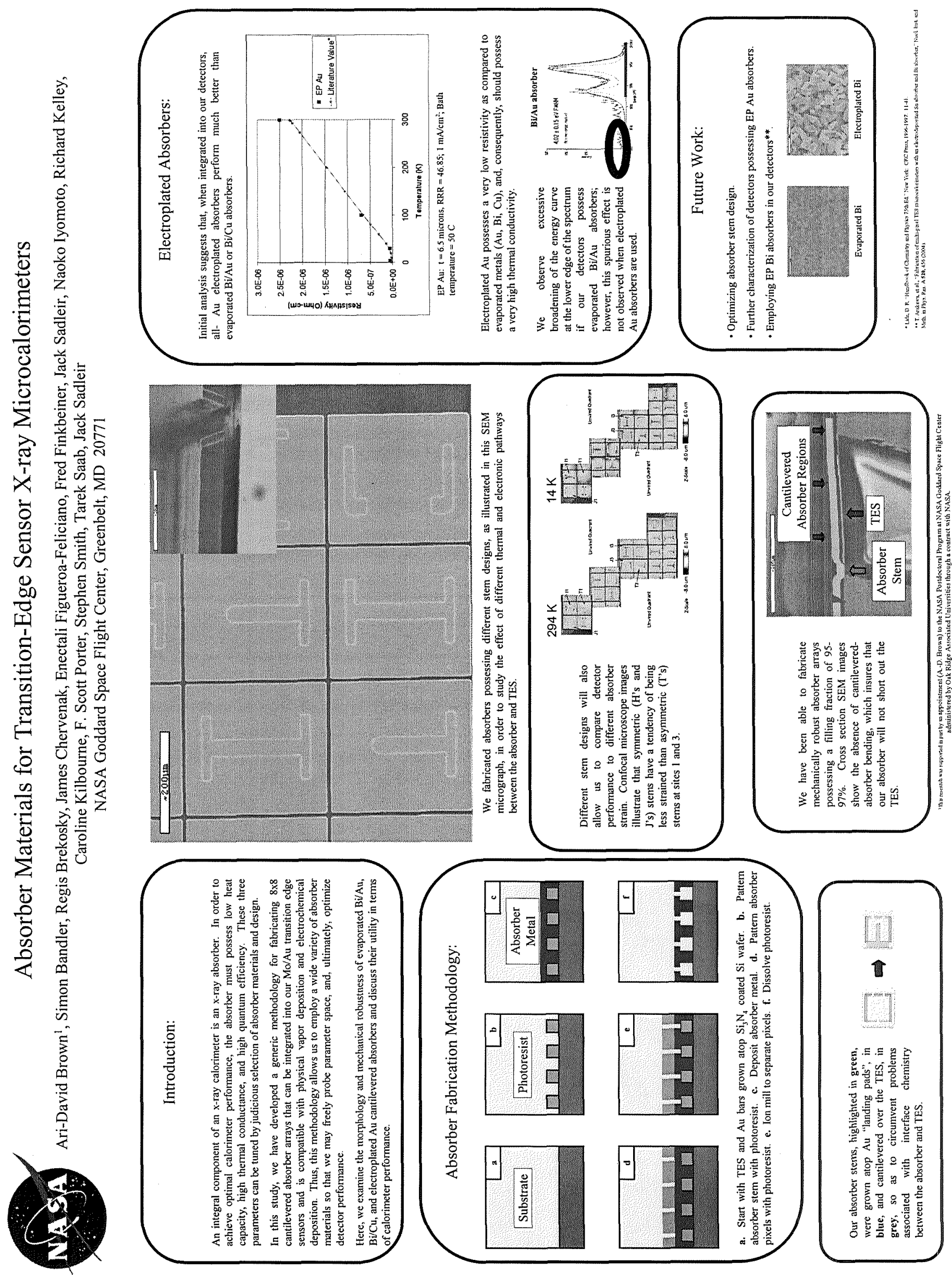

